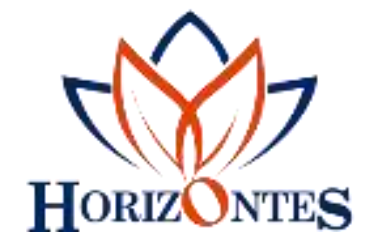

Horizontes. Revista de Investigación en Ciencias de la Educación Https://doi.org/10.33996/revistahorizontes.v5i17.168

Enero - marzo 2021

Volumen 5 / No. 17

ISSN: $2616-7964$

ISSN-L: 2616 - 7964

www.revistahorizontes.org pp. $207-229$

\title{
Gestión pedagógica y calidad educativa en una universidad pública del Perú
}

\author{
Pedagogical management and educational quality in a public university in Peru \\ Gestão pedagógica e qualidade educacional em uma universidade pública no Peru
}

ARTICULO DE INVESTIGACIÓN

\section{Yris Yolanda Bedoya Campos ${ }^{1}$ \\ ybedoyac@unia.edu.pe \\ ORCID: 0000-0003-0748-8830}
Edgardo Félix Palomino Torres ${ }^{2}$
edgardo.palomino@unh.edu.pe
ORCID: 0000-0002-4252-0704

\author{
Edgar Augusto Salinas Loarte ${ }^{2}$ \\ edgar.salinas@unh.edu.pe \\ ORCID: 0000-0003-4081-3834
}

\author{
Yuri Sánchez Solis ${ }^{2}$ \\ yuri.sanchez@unh.edu.pe \\ ORCID: 0000-0003-1300-728X
}

${ }^{1}$ Docente de la Facultad de Educación Intercultural y Humanidades de la Universidad Nacional Intercultural de la Amazonia

${ }^{2}$ Docente de la Facultad de Ciencias Empresariales de la Universidad Nacional de Huancavelica Recibido 15 de febrero 2021 | Arbitrado y aceptado 05 de marzo 2021| Publicado en marzo 2021

\section{RESUMEN}

El Objetivo general de la investigación es identificar la relación existente entre la gestión pedagógica y la calidad educativa en la Facultad de Educación y Ciencias Sociales de la Universidad Nacional de Ucayali. La metodología aplicada se basó en un diseño no experimental, de tipo de campo con niveles descriptivo, explicativo, analítico, sintético y estadístico. Los resultados se basaron en el análisis de los factores que influyen en la gestión pedagógica como fundamentación académica, organización e implementación, desarrollo curricular y supervisión y control de calidad. Como conclusión, la gestión pedagógica se relaciona negativamente con la calidad educativa en la Facultad de Educación y Ciencias Sociales de la Universidad Nacional de Ucayali, debido principalmente a dos factores: uno es que los docentes muestran desinterés en aplicar y analizar los documentos pertinentes para el desarrollo de los procesos de enseñanza-aprendizaje y el otro es que las autoridades no realizan o ejecutan de manera pertinente la gestión pedagógica para el desarrollo de una calidad educativa.

Palabras clave: Currículo; recursos; capacitación; gestión pedagógica; calidad educativa

\begin{abstract}
The general objective of the research is to identify the relationship between pedagogical management and educational quality in the Faculty of Education and Social Sciences of the National University of Ucayali. The applied methodology was based on a non-experimental, field-type design with descriptive, explanatory, analytical, synthetic and statistical levels. The results were based on the analysis of the factors that influence pedagogical management such as academic foundation, organization and implementation, curriculum development and supervision and quality control. In conclusion, pedagogical management is negatively related to educational quality in the Faculty of Education and Social Sciences of the National University of Ucayali, mainly due to two factors: one is that teachers show disinterest in applying and analyzing relevant documents for the development of the teaching-learning processes and the other is that the authorities do not carry out or execute in a pertinent way the pedagogical management for the development of an educational quality.
\end{abstract}

Key words: Curriculum; resources; training; pedagogical management; educational quality 


\section{RESUMO}

O objetivo geral da pesquisa é identificar a relação entre gestão pedagógica e qualidade educacional na Faculdade de Educação e Ciências Sociais da Universidade Nacional de Ucayali. A metodologia aplicada baseou-se em um delineamento não experimental do tipo de campo com níveis descritivo, explicativo, analítico, sintético e estatístico. Os resultados basearam-se na análise dos fatores que influenciam a gestão pedagógica, tais como formação acadêmica, organização e implementação, desenvolvimento e supervisão curricular e controle de qualidade. Em conclusão, a gestão pedagógica está negativamente relacionada à qualidade educacional na Faculdade de Educação e Ciências Sociais da Universidade Nacional de Ucayali, principalmente devido a dois fatores: um é que os professores mostram desinteresse em aplicar e analisar documentos relevantes para o desenvolvimento do ensino -processos de aprendizagem e o outro é que as autoridades não realizem ou executem de forma pertinente a gestão pedagógica para o desenvolvimento de uma qualidade educacional.

Palavras-chave: Currículo; recursos; treinamento; gestão pedagógica; qualidade educacional

\section{INTRODUCCIÓN}

La educación universitaria es uno de los pilares fundamentales de la sociedad porque brinda la posibilidad de superación de las personas formándolas para el mundo laboral y profesional. Esto a su vez permite el desarrollo y crecimiento económico y social de los países. En este sentido, la educación superior maneja mecanismos y/o políticas que permiten mejorar constantemente sus desarrollos académicos y administrativos. Esto es lo que se denomina gestión educativa. Por lo que, la gestión significa la acción o efecto de administrar (VISOR, 1999), y la gestión educativa se considera como un cuerpo organizado de principios $y$ regularidades se aplican para guiar y prescribir como han de funcionar y como se han de gestionar para cumplir con los propósitos y así conseguir la mayor eficacia y eficiencia posible (Guano Romero, 2014).

Es por ello, que la gestión pedagógica puede tener una relación con el fundamento de administrar las técnicas pedagógicas aplicadas en los procesos de enseñanza-aprendizaje. Antúnez (1996) tiene un concepto más específico, donde comprende un conjunto de acciones de movilización de recursos, personas, tiempo, dinero, materiales, entre otros elementos implica planificar actuaciones, distribuir tareas y responsabilidades, así como dirigir, coordinar y evaluar los procesos y sus resultados (López-Paredes, 2017).

Uno de los principales recursos de la gestión pedagógica son los docentes, donde su labor dependerá de las estrategias que debe aplicar para los procesos de enseñanzaaprendizaje. A su vez está influenciado por una serie de parámetros externos, como carácter socioeconómico y geográfico, que moldean principalmente a la motivación. En otras palabras, la gestión pedagógica del docente debe ir mucho más allá de reproducir y transmitir conocimientos; debe cumplir una función de transformación, de modo que enseñar no sea para él o ella un acto de transferencia mecánica de los saberes constituidos por otros. (Rey, 2014).

En este rol el docente es un mediador entre los alumnos y el contexto, su papel es orientar e incentivar a los estudiantes para que desarrollen competencias, con capacidades para interiorizar los diferentes elementos que interviene en el proceso educativo; el docente como mediador facilita la interacción para que el grupo participe en actividades de análisis y síntesis sustentadas en una acción reflexiva sobre lo realizado y lo que se puede realizar.

El docente, como mediador, tiene que estar en capacidad de proporcionar elementos conceptuales, procedimentales a los alumnos desde su posición de enseñar a pensar y aprender a aprender, a fin de apoyar en la construcción del conocimiento $\mathrm{y}$ en la 
realización de actividades que favorezcan el desarrollo del perfil de competencias esperados, todo esto, en función de las demandas que surgen de las múltiples y cambiantes situaciones del entorno, de esta forma participa en la configuración de procesos curriculares, dentro de metodologías integradoras $y$ especificas estrategias de aprendizaje.

Es así, que el sentido fundamental del trabajo y gestión pedagógica del docente es el desarrollo y formación del nuevo profesional con sentido de pertenencia social, en la búsqueda constante de las soluciones. Es decir, la gestión pedagógica parte de reconocer los componentes personales y no personales del proceso docente educativo, la cual se encuentran en estrecha correlación y tienen como propósito lograr la formación y desarrollo de la personalidad del estudiante como ser social (López-Paredes, 2017).

Además, la gestión pedagógica implica el manejo y desarrollo de actividades por parte de los directivos de las instituciones educativas. Según López (2010), señala que la gestión pedagógica del directivo es un proceso que conduce hacia el logro del mejoramiento curricular a través del desempeño docente; procurando buscar mejora e innovación curricular, el desarrollo profesional y personal del docente (citado por Pareja, 2020). Del mismo modo, Contreras (2016) comenta que la gestión pedagógica de los directivos debe:

Centrarse en la mejora de los aprendizajes significativos a través de los docentes quienes orientan y dirigen la planificación escolar, considerando estrategias para el trabajo pedagógico a desarrollarse en la institución educativa; los cuales tendrán repercusión en la mejora de los resultados académicos y por ende en la calidad educativa.(citado por Pareja, 2020, pág. 12).

Por lo cual, la gestión pedagógica se puede establecer a través de modelos que permitan una mejor interacción entre los factores que involucran el desarrollo académico de una institución. Sus orientaciones abarcan:

El desarrollo de la autonomía; promueve y estimula la permanente interacción con los actores internos y externos del aprendizaje; replantea la flexibilidad de la malla curricular en correspondencia a la visión antropocéntrica, con profundo respeto a concepciones filosóficas, espirituales, culturales y epistemológicas, ajustado a una formación humanista integral del estudiante, y propiciando el respeto por las diferencia individuales; transversa áreas vitales en el proceso de formación como la investigación, conocimiento de sí mismo, análisis del discurso, construcción y generación de conocimientos, tecnologías de información y comunicación. (Bracho-Pérez, 2018, pág. 207).

Los modelos de gestión pedagógica, según Chen-Quesada et al (2020), buscan apoyan tres principios que es la participación, la actitud frente al cambio y la innovación. Estas se describen en la Tabla 1 
Tabla 1. Principios de los modelos de gestión pedagógica.

\begin{tabular}{|c|c|}
\hline PRINCIPIOS & CARACTERÍSTICAS \\
\hline Participación & $\begin{array}{l}\text { La participación es una acción social dirigida a democratizar las } \\
\text { decisiones y que los resultados, producto de estas, sean compartidos y } \\
\text { valorados por la comunidad educativa, de manera que brinden } \\
\text { pertinencia e identidad. }\end{array}$ \\
\hline Actitud frente al cambio & $\begin{array}{l}\text { Se conceptualiza como aquellos comportamientos que enfrenta o vive } \\
\text { una comunidad educativa frente al cambio y que influyen positiva o } \\
\text { negativamente en el desarrollo o implementación de nuevos procesos } \\
\text { educativos. }\end{array}$ \\
\hline Innovación & $\begin{array}{l}\text { La innovación responde a una necesidad sentida en la comunidad } \\
\text { educativa y que conlleva la movilización de recursos y participación } \\
\text { colectiva, conducidos a la renovación o creación para las } \\
\text { transformaciones en materia curricular. Esta innovación puede } \\
\text { desarrollarse en los ámbitos de la gestión y la academia; persigue el } \\
\text { mejoramiento del desempeño docente, administrativo y del } \\
\text { estudiantado }\end{array}$ \\
\hline
\end{tabular}

Fuente: (Chen-Quesada et al, 2020)

Si esta situación se extrapola a la educación superior entonces hay presencia de una gestión pedagógica integradora. Aquí se articula los subsistemas, cultural académico, cultural investigativo y cultural laboral profesional, a partir de núcleos teóricos integradores que plantean procedimientos $y$ atienden las dimensiones instructiva, educativa, desarrolladora y administrativa, de forma articulada(Batista de los Ríos, 2017).

Esta situación, sin duda mejora la calidad educativa de la institución, de los docentes y de los estudiantes que están involucrados en estos desarrollos. Es así, que el término calidad cala en los estándares educativos en todos los niveles, en especial el superior. Según Cano (1998), definió calidad educativa como un proceso compartico de construcción continua más que como resultado, por lo que no es un estado estático sino flexible, por cuanto la institución educativa siempre deberá estar abocado a alcanzar más calidad(citado por Quispe Ramos, 2018). De la misma manera,
Schmelkes (1996) comenta que la calidad es considerar cuatro componentes fundamentales que deben observarse en un proceso educativo $\mathrm{y}$, sobre todo, en los resultados del mismo, los cuales son relevancia, eficacia, equidad y eficiencia(García Colina, Juárez Hernández, \& Salgado García, 2018).

Aunque, a pesar de estas percepciones realizadas por estos investigadores la gran mayoría de los mismo piensan que el concepto de calidad educativa es más amplio e involucra una serie de factores sociales, económicos, políticos y culturales, tanto del país, sociedad o institución educativa, por tal motivo cada institución presenta un concepto de calidad educativa acorde a sus alcances y limitaciones. En este sentido, según Calvo (2018) la calidad de una institución o de un programa se refiere a las características universales correspondientes a la educación superior en general, en las cuales va explicita la evaluación y el país tiene la obligación de garantizar que esa educación se entregue con calidad. Esto también lo comparte 
Quintana-Torres (2018) donde:

El mejoramiento de la calidad de la educación está, por lo tanto, condicionado al reconocimiento de las posibilidades reales que tienen las instituciones en el entorno sociocultural en el que se encuentran y al respeto por el proceso de maduración que debe tomar; de esta manera, cada institución puede concentrar acciones en aquello que sí puede hacer $\mathrm{y}$, gracias a ello, ganar la madurez que gradualmentele permita mover esos límites.(pág. 278)

Sin embargo, la madurez de la educación, en especial la superior se debe gracias al cumplimiento de ciertos factores básicos de la calidad de enseñanza universitaria, tal como se detalla en la Tabla 2.

En este contexto, se encuentra la educación superior peruana sumergida en insuficiencias que no le permiten generar la calidad que espera la sociedad. Dentro de esta se encuentra que la gestión pedagógica no está encaminada adecuadamente en el desarrollo docente; la política educativa no está de acorde con nuestra realidad nacional, de allí que se dan diversas dificultades para el desarrollo docente en el país, siendo deficiente e improvisado. Tal hecho nos impide obtener una educación de calidad y consecuentemente el rendimiento académico de los estudiantes se encontrará en un índice bajo.

Tabla 2. Factores básicos de la calidad de enseñanza universitaria.

\begin{tabular}{|c|c|}
\hline FACTORES & CARACTERÍSTICAS \\
\hline $\begin{array}{l}\text { Las actitudes, concepción } \\
\text { de la enseñanza y la } \\
\text { actuación del profesorado }\end{array}$ & $\begin{array}{l}\text { Considerar los principios pedagógicos, atención a los aprendizajes de } \\
\text { los estudiantes y a su interés por la asignatura, establecimiento de } \\
\text { estímulos para promover su participación, disponibilidad para } \\
\text { orientarles, buena comunicación con ellos, evaluación adecuada. }\end{array}$ \\
\hline $\begin{array}{l}\text { La competencia del } \\
\text { profesorado }\end{array}$ & $\begin{array}{l}\text { Nivel y actualidad de sus conocimientos teóricos y prácticos, capacidad } \\
\text { para su transmisión, dotes didácticos, formación continua. }\end{array}$ \\
\hline El plan de estudios & $\begin{array}{l}\text { Contenidos teóricos y prácticos, adecuación a los estudiantes y a las } \\
\text { demandas sociales de los correspondientes perfiles profesionales, } \\
\text { grado de operatividad. }\end{array}$ \\
\hline $\begin{array}{l}\text { Las infraestructuras y los } \\
\text { materiales }\end{array}$ & Instalaciones, equipos, materiales didácticos. \\
\hline $\begin{array}{l}\text { La organización de la } \\
\text { enseñanza }\end{array}$ & $\begin{array}{l}\text { Planificación detallada, distribución de los estudiantes entre los grupos, } \\
\text { adecuación de los horarios. }\end{array}$ \\
\hline La evaluación de la calidad & Que permita aprender de los errores y seguir mejorando. \\
\hline $\begin{array}{l}\text { La transparencia } \\
\quad \text { informativa }\end{array}$ & $\begin{array}{l}\text { En la institución, que facilitará la compartición del conocimiento y } \\
\text { generará confianza. }\end{array}$ \\
\hline $\begin{array}{l}\text { La participación de todos } \\
\text { los implicados }\end{array}$ & $\begin{array}{l}\text { Liderazgo participativo, clima de trabajo favorable, desarrollo y } \\
\text { crecimiento personal. }\end{array}$ \\
\hline
\end{tabular}

Fuente: (Bedoya Campos, Salinas Loarte, Palomino Torres y Sánchez Solís, 2021) 
Así mismo, el Estado peruano debe dar prioridades a las universidades del país dando mayor asignación presupuestaria. De igual forma, las universidades nacionales del país no cuentan con currículos integrales por facultades, el currículo debe establecer lineamientos comunes $\mathrm{y}$ un conjunto de aprendizajes obligatorios para todo el país, orientados a contribuir la identidad regional, nacional y universal. Dicho de otra forma, no estructuran un currículo acorde con las necesidades y realidad de cada facultad de las universidades; por tanto, no garantiza una calidad académica personal y social. Las universidades deben diseñar y ejecutar currículos de estudio que estén acorde a su realidad.

Pero lamentablemente en las universidades peruanas se desconoce estos pilares fundamentales de la docencia universitaria y se viene utilizando los llamados "métodos clásicos" de enseñanza, que permiten a los docentes conducir tradicionalmente el proceso enseñanza - aprendizaje de los estudiantes; entre los principales métodos clásicos, más usados, podemos señalar la clase expositiva, estudios de casos, seminarios, el estudio independiente supervisado, el laboratorio experimental, etc.

Con el fin de revertir esta dificultad y brindar alternativas de solución a este problema, la investigación está encaminada a identificar la relación existente entre la gestión pedagógica y la calidad educativa en la Facultad de Educación y Ciencias Sociales de la Universidad Nacional de Ucayali, con miras a realizar algunas sugerencias que puedan mejorar la gestión pedagógica en beneficio de una mejora de la calidad educativa. La metodología aplicada se basó en un diseño no experimental, de tipo de campo con niveles descriptivo, explicativo, analítico, sintético y estadístico.

\section{METODOLOGÍA}

El tipo de investigación es básico o descriptivo, porque en el estudio se utilizó conocimientos ya existentes sobre la gestión pedagógica. Los métodos de investigación utilizado es el descriptivo, explicativo, analítico, sintético y estadístico.

La investigación en referencia muestra una relación entre la variable independiente (gestión pedagógica) y la variable dependiente (calidad educativa). El nivel de investigación fue descriptivo correlacional, porque permite describió la situación real en que se encuentra la gestión pedagógica y la calidad educativa de carácter académico en la Universidad Nacional de Ucayali - Pucallpa.

La población es el conjunto de unidades de las que se desea obtener información y sobre las que van a generar las conclusiones, la cual puede ser definida como el conjunto finito o infinito de elementos, personas o cosas pertinentes a una investigación (Palella Stracuzzi y Martins Pestana, 2010). La población de estudio estuvo constituida por los docentes y alumnos de la Facultad de Educación y Ciencias Sociales de la Universidad Nacional de Ucayali, haciendo un total de 356 personas que comprende el 100\%. En la Tabla 3 se puede visualizar la distribución de la población docente y estudiantes. 
Tabla 3. Distribución de la población docente y estudiantes.

\begin{tabular}{ccccc}
\hline ESPECIALIDADES & ESTUDIANTES & DOCENTES & TOTAL & PORCENTAJE \\
\hline $\begin{array}{c}\text { Lengua y Literatura } \\
\text { Ciencias Sociales y Educación }\end{array}$ & 45 & 06 & 51 & 14,33 \\
$\begin{array}{c}\text { Intercultural } \\
\text { Ciencias Naturales y Medio }\end{array}$ & 29 & 08 & 37 & 10,39 \\
$\begin{array}{c}\text { Ambiente } \\
\text { Matemática, Física } \boldsymbol{A}\end{array}$ & 23 & 07 & 30 & 8,45 \\
$\begin{array}{c}\text { Informática } \\
\text { Primaria }\end{array}$ & 56 & 08 & 64 & 17,98 \\
TOTAL & 167 & 07 & 184 & 48,88 \\
\hline
\end{tabular}

Cuando las poblaciones son grandes, se impone la selección de los sujetos pues, por razones complejidad para el acopio de datos, sería imposible estudiarlos a todos, por lo que la muestra es una parte representativa cuyas características se reproduce de la manera más exacta posible (Palella Stracuzzi y Martins Pestana, 2010). Para determinar la muestra del número de docentes y alumnos por especialidad con que se trabajó, se hizo uso del muestreo aleatorio simple para proporciones, tal como se declive a continuación:

$$
n=\frac{Z^{2} x P x Q x N}{e^{2}(N-1)+Z^{2} x P x Q}
$$

Entonces;

$$
\begin{gathered}
n=\frac{(1,96)^{2} \times 0,25 \times 356}{(0,05)^{2}(356-1)+(1,96)^{2} \times 0,25} \\
n=\frac{341,9024}{1,8479} \\
n=185,0221 \approx 185
\end{gathered}
$$

Como la muestra es de 185 personas entonces fue distribuida por los estratos de especialidad. Para ello, se utilizó la ecuación siguiente:

$$
F R=\frac{n}{N}=0,52
$$

Entonces, esta fracción muestra se multiplicó por cada distribución de estudiantes y docentes que se visualizan en la Tabla 3, arrojando la muestra definitiva por especialidad tal como se observa en la Tabla 4. 
Tabla 4. Distribución de la muestra docente y estudiantes.

\begin{tabular}{ccccc}
\hline ESPECIALIDADES & ESTUDIANTES & DOCENTES & TOTAL & PORCENTAJE \\
\hline $\begin{array}{c}\text { Lengua y Literatura } \\
\text { Ciencias Sociales y Educación }\end{array}$ & 23 & 3 & 26 & 14 \\
$\begin{array}{c}\text { Intercultural } \\
\text { Ciencias Naturales y Medio } \\
\text { Ambiente }\end{array}$ & 15 & 4 & 19 & 10 \\
$\begin{array}{c}\text { Matemática, Física } \\
\text { Informática } \\
\text { Primaria } \\
\text { TOTAL }\end{array}$ & 29 & 4 & 16 & 9 \\
& 87 & 4 & 33 & 18 \\
\hline
\end{tabular}

La encuesta fue la técnica permitió aplicar un conjunto de preguntas concretas de opinión específica de los docentes y estudiantes para saber el nivel que tenían y otros datos. Además, se utilizó la observación como técnica directa y planificada, pues estuvo dirigida a la apreciación y recolección de datos a través de una observación natural, que sirvió para la observación de los hechos a estudiarse. Esto se materializó utilizando el instrumento de la guía de observación.

El cuestionario se estructuró en una serie de preguntas de tal modo que se pudo recolectar las informaciones necesarias para el desarrollo de la investigación. Se formularon interrogantes a los estudiantes. El cuestionario utilizado para aplicar a la muestra se observa en la Tabla 5.

Tabla 5. Preguntas del cuestionario para medir la gestión pedagógica y la calidad educativa de Universidad Nacional de Ucayali.

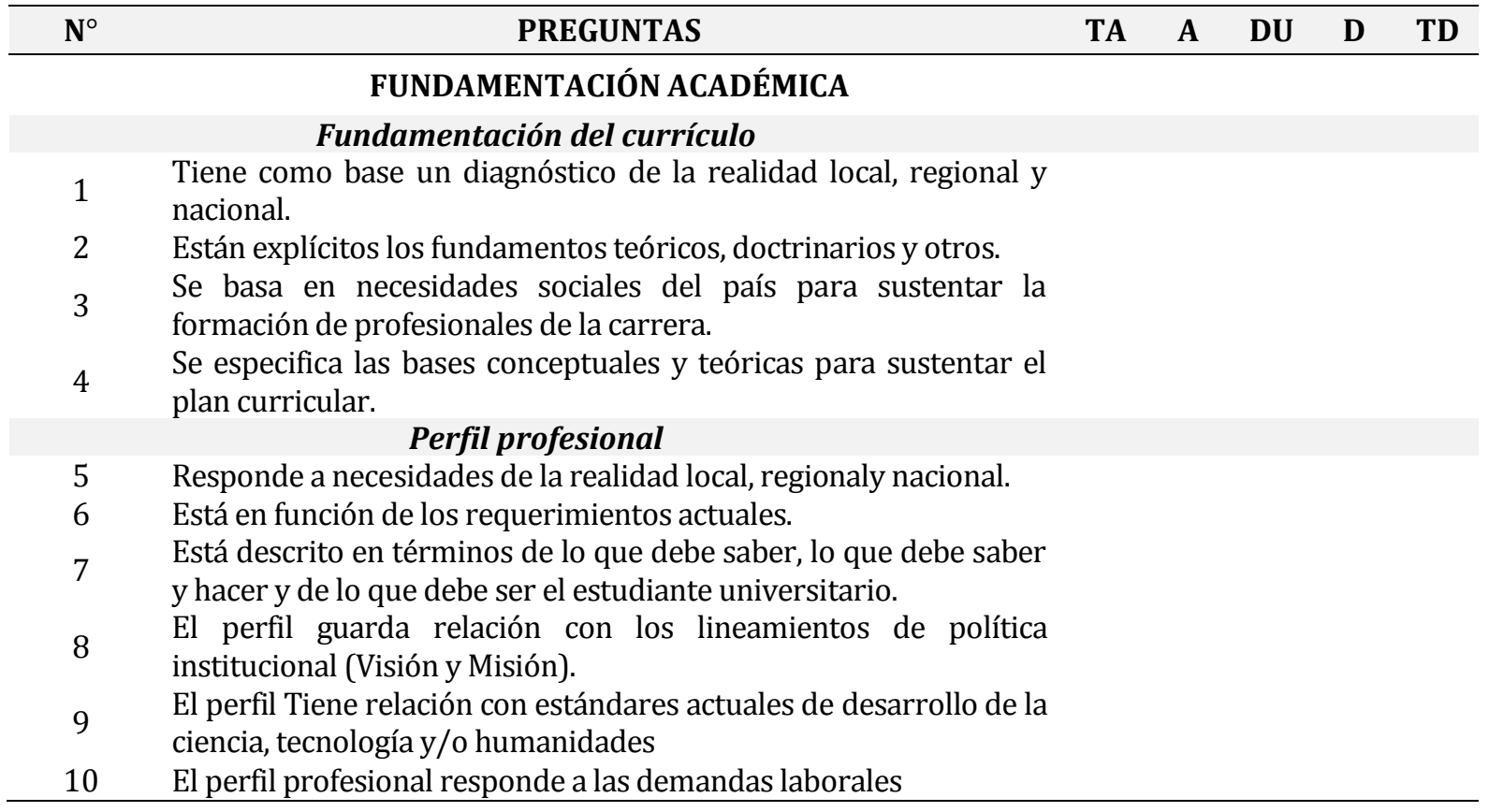




\begin{tabular}{|c|c|c|c|c|c|c|}
\hline $\mathbf{N}^{\circ}$ & PREGUNTAS & TA & A & DU & D & TD \\
\hline 11 & Existe coherencia con los objetivos de las asignaturas. & & & & & \\
\hline 12 & $\begin{array}{l}\text { El perfil contribuye a la acreditación y certificación de la } \\
\text { calidad educativa }\end{array}$ & & & & & \\
\hline & Plan de estudio & & & & & \\
\hline 13 & Presenta una estructura de organización de asignaturas. & & & & & \\
\hline 14 & La asignación de créditos por asignatura guarda relación con el & & & & & \\
\hline 15 & Los prerrequisitos tienen relación con las asignaturas & & & & & \\
\hline 16 & $\begin{array}{l}\text { Las asignaturas responden a las exigencias de los avances de la } \\
\text { ciencia y especialidad. }\end{array}$ & & & & & \\
\hline 17 & $\begin{array}{l}\text { El plan de estudios contiene lo suficiente en cuanto a número } \\
\text { de asignatura y crédito }\end{array}$ & & & & & \\
\hline 18 & $\begin{array}{l}\text { Aplica criterios de: Secuencialidad y Continuidad } \\
\text { ORGANIZACIÓN E IMPLEMENTACIóN }\end{array}$ & & & & & \\
\hline & Personal docente y preparación & & & & & \\
\hline 19 & $\begin{array}{l}\text { Parte de un diagnóstico de la realidad y de las necesidades del } \\
\text { potencial humano. }\end{array}$ & & & & & \\
\hline 20 & Determina el número de docentes requeridos. & & & & & \\
\hline 21 & Especifica las necesidades por asignaturas y/o actividades. & & & & & \\
\hline 22 & $\begin{array}{l}\text { Especifica las características y nivel de preparación deseable } \\
\text { de docentes. }\end{array}$ & & & & & \\
\hline 23 & $\begin{array}{l}\text { Considera eventos de capacitación para el personal docente y } \\
\text { administrativo. }\end{array}$ & & & & & \\
\hline & Recursos físicos & & & & & \\
\hline 24 & $\begin{array}{l}\text { Equipos y mobiliarios de escuela satisface } \\
\text { Necesidades para la implementación del currículo. }\end{array}$ & & & & & \\
\hline 25 & Las instalaciones físicas de la facultad/escuela son suficientes. & & & & & \\
\hline Recur & Administrativos & & & & & \\
\hline 26 & $\begin{array}{l}\text { El apoyo al desarrollo curricular que brinda el sistema } \\
\text { administrativo es óptimo }\end{array}$ & & & & & \\
\hline 27 & $\begin{array}{l}\text { El personal administrativo cumple con la misión universitaria } \\
\text { y contribuye al desarrollo del currículo. }\end{array}$ & & & & & \\
\hline & Recursos Didácticos y Materiales & & & & & \\
\hline 28 & Contribuyen al desarrollo curricular. & & & & & \\
\hline 29 & Propician las innovaciones. & & & & & \\
\hline 30 & Los recursos audiovisuales son suficientes. & & & & & \\
\hline 31 & Existe uso racional de los recursos didácticos. & & & & & \\
\hline 32 & El equipamiento de la biblioteca es racionalmente suficiente. & & & & & \\
\hline 33 & El sistema de internet apoya al desarrollo del currículo. & & & & & \\
\hline 34 & $\begin{array}{l}\text { El número de computadoras conectadas al sistema de internet } \\
\text { satisface los requerimientos. }\end{array}$ & & & & & \\
\hline & DESARROLLO CURRICULAR & & & & & \\
\hline & Balance en las áreas curriculares & & & & & \\
\hline 35 & $\begin{array}{l}\text { Presenta un cuadro correcto de distribución de áreas curriculares, } \\
\text { con horas y créditos asignados a cada uno de ellos. }\end{array}$ & & & & & \\
\hline 36 & $\begin{array}{l}\text { Existen especificaciones del peso asignado a cada área del } \\
\text { currículo. }\end{array}$ & & & & & \\
\hline
\end{tabular}




\begin{tabular}{|c|c|c|c|c|c|c|}
\hline $\mathbf{N}^{\circ}$ & PREGUNTAS & TA & A & DU & D & TD \\
\hline 37 & $\begin{array}{l}\text { La distribución de áreas horas y crédito responden al modelo } \\
\text { curricular propuesto. }\end{array}$ & & & & & \\
\hline 38 & $\begin{array}{l}\text { Hay un nivel de aproximación entre el peso deseable } \\
\text { establecido por el modelo para cada una de las áreas. }\end{array}$ & & & & & \\
\hline & Equilibrio entre la teoría y la práctica & & & & & \\
\hline 39 & $\begin{array}{l}\text { Especifica el énfasis teórico y práctico de cada asignatura o } \\
\text { actividad. }\end{array}$ & & & & & \\
\hline 40 & $\begin{array}{l}\text { Determina el número de horas teóricas y el número de horas } \\
\text { prácticas de cada asignatura o actividad en relación a los créditos } \\
\text { signados. }\end{array}$ & & & & & \\
\hline & Índice ponderado de prerrequisitos & & & & & \\
\hline 41 & $\begin{array}{l}\text { El número de materias preladas por semestre se encuentran } \\
\text { en el rango aceptable (mínimo } 25 \% \text { y máximo } 35 \% \text {. }\end{array}$ & & & & & \\
\hline 42 & Se han invitado las prelaciones innecesarias. & & & & & \\
\hline 43 & Se presenta el flujo grama de prelaciones (malla curricular) & & & & & \\
\hline & Racionalización del tiempo curricular & & & & & \\
\hline 44 & $\begin{array}{l}\text { Se especifica el trabajo docente, la preparación de clases prácticas } \\
\text { y/o actividades, la asesoría, consejería y los momentos para la } \\
\text { investigación. }\end{array}$ & & & & & \\
\hline 45 & $\begin{array}{l}\text { Establece la organización del currículo integral, cuadro lógico } \\
\text { (secuencia progresiva de los contenidos por áreas y especialidad. } \\
\text { SUPERVISIÓN Y CONTROL DE CALIDAD }\end{array}$ & & & & & \\
\hline & Supervisión académica & & & & & \\
\hline 46 & $\begin{array}{l}\text { Se ha previsto el asesoramiento técnico científico ydidáctico } \\
\text { para la actualización. }\end{array}$ & & & & & \\
\hline 47 & Existe disponible el cronograma de las actividades académicas. & & & & & \\
\hline 48 & Existe un registro de seguimiento al estudiante y los egresados. & & & & & \\
\hline 49 & $\begin{array}{l}\text { Se ha cumplido con el cronograma de capacitación a docentes y } \\
\text { personal administrativo }\end{array}$ & & & & & \\
\hline & Pertinencia & & & & & \\
\hline 50 & $\begin{array}{l}\text { El modelo curricular está en concordancia con las necesidades } \\
\text { y expectativas de la comunidad. }\end{array}$ & & & & & \\
\hline 51 & $\begin{array}{l}\text { Plantea acciones de desarrollo sostenible del sistema y los } \\
\text { ecosistemas del entorno. }\end{array}$ & & & & & \\
\hline 52 & $\begin{array}{l}\text { Las áreas del currículo corresponden al perfil que propone el } \\
\text { modelo. }\end{array}$ & & & & & \\
\hline 53 & $\begin{array}{l}\text { Existe relación entre el plan curricular y los ejes de interacción } \\
\text { del modelo propuesto. }\end{array}$ & & & & & \\
\hline 54 & Existe aceptación del currículo desarrollado en el contexto. & & & & & \\
\hline 55 & $\begin{array}{l}\text { Existen políticas de colocación de los egresados en el mercado } \\
\text { ocupacional. }\end{array}$ & & & & & \\
\hline 56 & Los egresados tienen acogida en el mercado ocupacional. & & & & & \\
\hline \multicolumn{7}{|c|}{ LEYENDA: } \\
\hline & TA = Totalmente de acuerdo & & & & & \\
\hline & $\mathbf{A}=$ Acuerdo & & & & & \\
\hline & DU $=$ Duda & & & & & \\
\hline \multirow{2}{*}{\multicolumn{7}{|c|}{$\begin{array}{r}\mathbf{D}=\text { Desacuerdo } \\
\text { TD }=\text { Totalmente en desacuerdo }\end{array}$}} \\
\hline & almente en desacuerdo & & & & & \\
\hline
\end{tabular}


Se usó la ficha de observación para registrar información empírica de las dimensiones infraestructura educativa, medios y materiales educativos e implementación de tecnología e informática (VD), su administración estuvo a cargo del investigador.

\section{RESULTADOS Y DISCUSIÓN}

En la Tabla 6 se puede visualizar los resultados de la fundamentación académica correspondiente a la fundamentación del currículo.

Tabla 6. Resultados sobre Fundamentación del Currículo aplicada a la muestra de la Universidad Nacional de Ucayali.

\begin{tabular}{|c|c|c|c|c|c|c|}
\hline \multicolumn{7}{|c|}{$\begin{array}{l}\text { FUNDAMENTACIÓN ACADÉMICA } \\
\text { Fundamentación del currículo }\end{array}$} \\
\hline $\mathbf{N}^{\circ}$ & PREGUNTAS & $\begin{array}{l}\text { TA } \\
\%\end{array}$ & $\begin{array}{l}\text { A } \\
\%\end{array}$ & $\begin{array}{l}\text { DU } \\
\%\end{array}$ & $\begin{array}{l}\text { D } \\
\%\end{array}$ & $\begin{array}{l}\text { TD } \\
\%\end{array}$ \\
\hline 1 & Tiene como base un diagnóstico de la realidad local, regional y nacional. & 0 & 25 & 25 & 50 & 0 \\
\hline 2 & Están explícitos los fundamentos teóricos, doctrinarios y otros. & 0 & 75 & 0 & 25 & 0 \\
\hline 3 & $\begin{array}{l}\text { Se basa en necesidades sociales del país para sustentar la formación de } \\
\text { profesionales de la carrera. }\end{array}$ & 0 & 0 & 50 & 25 & 25 \\
\hline 4 & $\begin{array}{l}\text { Se especifica las bases conceptuales y teóricas para sustentar el plan } \\
\text { curricular. }\end{array}$ & 0 & 75 & 0 & 0 & 25 \\
\hline
\end{tabular}

La muestra del estudio consideró que la fundamentación del currículo tiene un 50\% de desacuerdo en que se base en un diagnóstico local, regional y nacional, debido a que pueden considerar que depende de la carrera que se va a estudiar y los objetivos que persigue sin considerar los factores sociales, económicos o culturales. Esto lo comprueba el $75 \%$ de la muestra consideró que la fundamentación es teórica, doctrinaria, entre otros., así mismo lo refiere al 50\% de dudas que consideró el factor social para la formación de la carrera. Del mismo modo, el $75 \%$ de la muestra sostuvo que el plan curricular se sustenta por las bases teóricas y conceptuales.

En la Tabla 7 se muestra los resultados de la encuesta en relación al perfil profesional en la fundamentación académica. 
Tabla 7. Resultados sobre perfil profesional aplicada a la muestra de la Universidad Nacional de Ucayali.

\begin{tabular}{|c|c|c|c|c|c|c|}
\hline \multicolumn{7}{|c|}{$\begin{array}{l}\text { FUNDAMENTACIÓN ACADÉMICA } \\
\text { Perfil profesional }\end{array}$} \\
\hline \multirow{2}{*}{$\mathbf{N}^{\circ}$} & \multirow{2}{*}{ PREGUNTAS } & TA & $\mathbf{A}$ & DU & D & TD \\
\hline & & $\%$ & $\%$ & $\%$ & $\%$ & $\%$ \\
\hline 5 & Responde a necesidades de la realidad local, regional y nacional. & 0 & 25 & 50 & 25 & 0 \\
\hline 6 & Está en función de los requerimientos actuales. & 0 & 50 & 0 & 50 & 0 \\
\hline 7 & $\begin{array}{l}\text { Está descrito en términos de lo que debe saber, lo que debe saber y hacer y } \\
\text { de lo que debe ser el estudiante universitario. }\end{array}$ & 0 & 25 & 50 & 25 & 0 \\
\hline 8 & $\begin{array}{l}\text { El perfil guarda relación con los lineamientos de política institucional } \\
\text { (Visión y Misión). }\end{array}$ & 0 & 0 & 75 & 25 & 0 \\
\hline 9 & $\begin{array}{l}\text { El perfil tiene relación con estándares actuales de desarrollo de la ciencia, } \\
\text { tecnología y/o humanidades }\end{array}$ & 0 & 0 & 100 & 0 & 0 \\
\hline 10 & El perfil profesional responde a las demandas laborales & 0 & 25 & 75 & 0 & 0 \\
\hline 11 & Existe coherencia con los objetivos de las asignaturas. & 0 & 75 & 0 & 25 & 0 \\
\hline 12 & El perfil contribuye a la acreditación y certificación de la calidad educativa & 0 & 50 & 25 & 25 & 0 \\
\hline
\end{tabular}

El $50 \%$ de la muestra señaló que tiene dudas si el perfil profesional responde a las necesidades locales regionales o nacionales, aunque muestran que existe paridad entre de acuerdo y desacuerdo si está en función de los requerimientos actuales. También presentaron dudas, en un $50 \%$, si esta descrito en función del estudiante universitario, de la misma manera presenta un $75 \%$ de dudas si cumplió con los lineamientos de la política institucional, así mismo la totalidad de la muestra manifestó que hay dudas con respecto a la relación que existe el perfil profesional y los estándares de desarrollo de la ciencia. También, la muestra de estudio señaló que tiene dudas, en un $75 \%$, si el perfil profesional responde a las demandas laborales, pero hay un $75 \%$ que está de acuerdo con que cumple con las especificaciones y objetivos de las asignaturas. Del mismo modo, el $50 \%$ comentaron que el perfil contribuye a la acreditación y certificación de la calidad educativa.

Por lo cual, la mayoría de las respuestas arrojadas en el perfil profesional muestran dudas, lo que se manifiesta que dentro de la institución no se cumple con la comunicación organizacional, donde cada miembro de la misma este identificado con los objetivos, visión y misión de la universidad. La comunicación organización debe ser parte importante dentro de la gestión pedagógica.

Al mismo tiempo, los resultados en función del plan de estudio están reflejados en la Tabla 8. 
Tabla 8. Resultados sobre plan de estudios aplicada a la muestra de la Universidad Nacional de Ucayali.

\begin{tabular}{|c|c|c|c|c|c|c|}
\hline \multicolumn{7}{|c|}{ FUNDAMENTACIÓN ACADÉMICA } \\
\hline $\mathbf{N}^{\circ}$ & PREGUNTAS & TA & $\mathbf{A}$ & DU & $\mathbf{D}$ & TD \\
\hline Iv & 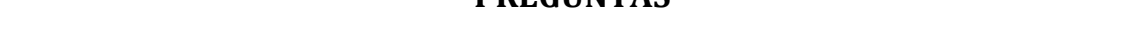 & $\%$ & $\%$ & $\%$ & $\%$ & $\%$ \\
\hline 13 & Presenta una estructura de organización de asignaturas. & 0 & 25 & 75 & 0 & 0 \\
\hline 14 & $\begin{array}{l}\text { La asignación de créditos por asignatura guarda relación con el } \mathrm{N}^{\circ} \text { de horas } \\
\text { teóricas y prácticas. }\end{array}$ & 0 & 75 & 25 & 0 & 0 \\
\hline 15 & Los prerrequisitos tienen relación con las asignaturas & 0 & 100 & 0 & 0 & 0 \\
\hline 16 & $\begin{array}{l}\text { Las asignaturas responden a las exigencias de los avances de la ciencia y } \\
\text { especialidad. }\end{array}$ & 0 & 0 & 75 & 25 & 0 \\
\hline 17 & $\begin{array}{l}\text { El plan de estudios contiene lo suficiente en cuanto a número de asignatura } \\
\text { y crédito }\end{array}$ & 0 & 50 & 25 & 25 & 0 \\
\hline 18 & Aplica criterios de: Secuencialidad y Continuidad & 0 & 100 & 0 & 0 & 0 \\
\hline
\end{tabular}

La muestra de estudio muestra que existe un $75 \%$ de dudas en que las asignaturas presentan una estructura de organización en el pan de estudios. Por otra parte, el $75 \%$ consideró que está de acuerdo que la asignación de créditos cumple con las horas de teoría y práctica. Así mismo, el 100\% está de acuerdo con que los prerrequisitos están en relación con las asignaturas. En cambio, hay un $75 \%$ de dudas en que las asignaturas responden a las exigencias de los avances de las ciencias y tecnologías. Por otro lado, el $50 \%$ está de acuerdo con que el plan de estudios contiene suficiente entre el número de asignaturas y créditos, de la misma manera la totalidad de los encuestados manifiestan que estás de acuerdo que el plan de estudios aplica secuencialidad y continuidad.

En consecuencia, se sigue manifestando problemas en la comunicación organizacional, donde la universidad manifieste cual es la finalidad que cumplen las asignaturas y sus créditos en función del plan de estudios de las carreras que hacen vida dentro de la facultad. Sin embargo, los encuestados manifiestan que están de acuerdo en aquellas situaciones que perciben de primera mano cómo los prerrequisitos de las asignaturas y la asignación de créditos en relación con las horas teórica-práctica.

En la Tabla 9 se visualiza los resultados del personal docente y preparación que depende del factor organización e implementación en el estudio. 
Tabla 9. Resultados sobre personal docente y preparación aplicada a la muestra de la Universidad Nacional de Ucayali.

\begin{tabular}{|c|c|c|c|c|c|c|}
\hline \multicolumn{7}{|c|}{$\begin{array}{l}\text { ORGANIZACIÓN E IMPLEMENTACIÓN } \\
\text { Personal docente y preparación }\end{array}$} \\
\hline \multirow{2}{*}{$\mathbf{N}^{\circ}$} & \multirow{2}{*}{ PREGUNTAS } & TA & $\mathbf{A}$ & DU & D & TD \\
\hline & & $\%$ & $\%$ & $\%$ & $\%$ & $\%$ \\
\hline 19 & $\begin{array}{l}\text { Parte de un diagnóstico de la realidad } \\
\text { y de las necesidades del potencial }\end{array}$ & 0 & 25 & 50 & 25 & 0 \\
\hline 20 & $\begin{array}{l}\text { Determina el número de docentes } \\
\text { requeridos. }\end{array}$ & 25 & 25 & 25 & 25 & 0 \\
\hline 21 & $\begin{array}{l}\text { Especifica las necesidades por } \\
\text { asignaturas y/o actividades. }\end{array}$ & 0 & 75 & 25 & 0 & 0 \\
\hline 22 & $\begin{array}{l}\text { Especifica las características y nivel de } \\
\text { preparación deseable de docentes. }\end{array}$ & 0 & 50 & 50 & 0 & 0 \\
\hline 23 & $\begin{array}{l}\text { Considera eventos de capacitación } \\
\text { para el personal docente y } \\
\text { administrativo. }\end{array}$ & 0 & 0 & 50 & 25 & 25 \\
\hline
\end{tabular}

Existen un $50 \%$ de dudas en que los docentes realizaban un diagnóstico de la realidad y de las necesidades del potencial humano, según la percepción de la muestra estudio. No hubo un consenso exacto en los encuestados referente a que la institución determina el número de docentes requeridos por especialidad, así mismo con las características y nivel de preparación deseables de los mismos. El 75\% de la muestra estuvo de acuerdo con que los docentes especifican las necesidades por asignaturas y/o actividades. El 50\% tuvo dudas si la universidad considera importante la capacitación para el personal docente y administrativo.

La gestión pedagógica en la institución de educación superior no considera como factor clave el personal docente y su preparación para las asignaturas que corresponden a cada especialidad. Un parámetro importante en el desarrollo de la calidad educativa es el tipo de docente y la preparación que tiene para afrontar las actividades tecnopedagógicas que puedan acontecer en el desarrollo de los contenidos programáticos de las asignaturas. La institución debe planificar estrategias de capacitación para brindar un efecto colateral en el rendimiento académico de los estudiantes, pero a su vez fortalecerá la motivación de los docentes en el desarrollo académico dentro del aula de clase.

Los resultados sobre los recursos físicos necesario en el desarrollo de la Organización e implementación que se obtuvieron en la encuesta se observan en la Tabla 10. 
Tabla 10. Resultados sobre recursos físicos aplicada a la muestra de la Universidad Nacional de Ucayali.

\begin{tabular}{|c|c|c|c|c|c|c|}
\hline \multicolumn{7}{|c|}{$\begin{array}{l}\text { ORGANIZACIÓN E IMPLEMENTACIÓN } \\
\text { Recursos físicos }\end{array}$} \\
\hline \multirow{2}{*}{$\mathbf{N}^{\circ}$} & \multirow{2}{*}{ PREGUNTAS } & TA & $\mathbf{A}$ & DU & D & TD \\
\hline & & $\%$ & $\%$ & $\%$ & $\%$ & $\%$ \\
\hline 24 & $\begin{array}{l}\text { Equipos y mobiliarios de escuela satisface necesidades para la } \\
\text { implementación del currículo. }\end{array}$ & 0 & 25 & 50 & 25 & 0 \\
\hline 25 & Las instalaciones físicas de la facultad/escuela son suficientes. & 0 & 0 & 50 & 50 & 0 \\
\hline
\end{tabular}

El 50\% de la muestra reflejó que tienen dudas si los equipos e inmobiliarios de la institución satisface las necesidades para la implementación del currículo. En cambio, no hubo consenso entre los encuestados si las instalaciones físicas de la facultad son suficientes para el desarrollo de las diversas actividades académicas. Las respuestas a estas dos preguntas no dejan claro en qué condiciones esta la infraestructura de la universidad, pero lo que si es que una buena disposición de los mismos es fundamental para el desarrollo de los contenidos de cada asignatura. Al existir duda entre la muestra de estudio refleja que la institución no está apostando en el desarrollo de espacios acordes a los tiempos presentes, es necesario que se ejecute una gestión pedagógica enfocada en el desarrollo de estos recursos para que no baje la calidad educativa en la organización.

En la Tabla 11 muestra los resultados de los recursos administrativos aplicada a la muestra de la investigación.

Tabla 11. Resultados sobre recursos administrativos aplicada a la muestra de la Universidad Nacional de Ucayali.

\begin{tabular}{clccccc}
\hline \multicolumn{7}{c}{ ORGANIZACIÓN E IMPLEMENTACIÓN } \\
\multicolumn{1}{c}{ Recursos físicos } \\
\hline $\mathbf{N}^{\circ}$ & \multicolumn{1}{c}{ TA } & A & DU & D & TD \\
PREGUNTAS & $\%$ & $\%$ & $\%$ & $\%$ & $\%$ \\
26 & $\begin{array}{l}\text { El apoyo al desarrollo curricular que } \\
\text { brinda el sistema administrativo es } \\
\text { óptimo }\end{array}$ & 0 & 0 & 75 & 0 & 25 \\
$\begin{array}{l}\text { El personal administrativo cumple con } \\
\text { la misión universitaria y contribuye al } \\
\text { desarrollo del currículo. }\end{array}$ & 0 & 25 & 50 & 25 & 0 \\
\hline
\end{tabular}


En la encuesta realizada a la muestra de estudio el 50\% manifestó duda si el personal administrativo cumplía con la misión universitaria y contribuye al desarrollo del currículo, así como también muestran que tienen un $75 \%$ de dudas de que el sistema administrativo apoya de manera óptima al desarrollo curricular. Ambos resultados, arrojaron que no hay un vínculo entre la parte administrativa con el sistema académico, por lo que su desempeño no tiene importancia en el desarrollo curricular. Esto no permite una vinculación con la misión y visión de la institución. La gestión pedagógica debe buscar entrelazar la funcionabilidad del personal administrativo con el desarrollo académico debido a que muchos de los trámites son ejecutados por este personal lo que produciría un incremento en la productividad del trabajo.

Los resultados de los recursos didácticos y materiales presentes en la organización e implementación se visualizan en la Tabla 12.

Tabla 12. Resultados sobre recursos didácticos y materiales aplicada a la muestra de la Universidad Nacional de Ucayali.

\begin{tabular}{|c|c|c|c|c|c|c|}
\hline \multicolumn{7}{|c|}{$\begin{array}{c}\text { ORGANIZACIÓN E IMPLEMENTACIÓN } \\
\text { Recursos didácticos y materiales }\end{array}$} \\
\hline \multirow{2}{*}{$\mathbf{N}^{\circ}$} & \multirow{2}{*}{ PREGUNTAS } & TA & $\mathbf{A}$ & DU & D & TD \\
\hline & & $\%$ & $\%$ & $\%$ & $\%$ & $\%$ \\
\hline 28 & Contribuyen al desarrollo curricular. & 0 & 0 & 100 & 0 & 0 \\
\hline 29 & Propician las innovaciones. & 0 & 0 & 100 & 0 & 0 \\
\hline 30 & Los recursos audiovisuales son suficientes. & 0 & 0 & 25 & 75 & 0 \\
\hline 31 & Existe uso racional de los recursos didácticos. & 0 & 0 & 25 & 75 & 0 \\
\hline 32 & El equipamiento de la biblioteca es racionalmente suficiente. & 0 & 25 & 25 & 50 & 0 \\
\hline 33 & El sistema de internet apoya al desarrollo del currículo. & 0 & 50 & 25 & 0 & 25 \\
\hline 34 & $\begin{array}{l}\text { El número de computadoras conectadas al sistema de internet satisface los } \\
\text { requerimientos. }\end{array}$ & 0 & 0 & 50 & 25 & 25 \\
\hline
\end{tabular}

Los resultados arrojan que el $100 \%$ de los encuestados tienen dudas con respecto a la que los recursos didácticos y materiales contribuyen al desarrollo curricular y propician las innovaciones. En cambio, el $75 \%$ manifestó que están en desacuerdo que los recursos audiovisuales son suficientes, así como si existe un uso racional de los recursos didácticos. Así mismo, el 50\% estuvo en desacuerdo en que el equipo de la biblioteca es racionalmente suficiente. Por otra parte, el $50 \%$ comenta que el sistema de internet presta el apoyo al desarrollo del currículo. Del mismo modo, el 50\% presentaron dudas si la cantidad de computadoras conectadas a internet satisfacen a los requerimientos. En definitiva, la encuesta demuestra que no existe apoyo en los recursos y herramientas 
pedagógicas utilizadas por los docentes, tanto en materia de clases presenciales como en clases virtuales. Es importante que la institución demuestre apoyo a los recursos pedagógicos con el fin de poder aumentar la calidad educativa, pero sobre todo en el aumento del rendimiento académico.

En la Tabla 13 se detalla los resultados del balance en las áreas curriculares según el desarrollo curricular que se obtuvieron en la encuesta realizada a la muestra estudio.

Tabla 13. Resultados sobre balance en las áreas curriculares aplicada a la muestra de la Universidad Nacional de Ucayali.

\begin{tabular}{|c|c|c|c|c|c|c|}
\hline \multicolumn{6}{|c|}{ DESARROLLO CURRICULAR } & \\
\hline $\mathbf{N}^{\circ}$ & DDEGUUTAS & TA & A & DU & D & TD \\
\hline IN & PKEGUN I AS & $\%$ & $\%$ & $\%$ & $\%$ & $\%$ \\
\hline 35 & $\begin{array}{l}\text { Presenta un cuadro correcto de distribución de áreas curriculares, con horas } \\
\text { y créditos asignados a cada uno de ellos. }\end{array}$ & 0 & 75 & 0 & 25 & 0 \\
\hline 36 & Existen especificaciones del peso asignado a cada área del currículo. & 0 & 50 & 50 & 0 & 0 \\
\hline 37 & $\begin{array}{l}\text { La distribución de áreas horas y crédito responden al modelo curricular } \\
\text { propuesto. }\end{array}$ & 0 & 100 & 0 & 0 & 0 \\
\hline 38 & $\begin{array}{l}\text { Hay un nivel de aproximación entre el peso deseable establecido por el } \\
\text { modelo para cada una de las áreas. }\end{array}$ & 0 & 50 & 50 & 0 & 0 \\
\hline
\end{tabular}

Los resultados muestran que el $75 \%$ de los encuestados están de acuerdo con que hay una distribución correcta de las áreas curriculares en función de las horas y créditos asignados. También, el 100\% manifestó que está de acuerdo a la distribución de áreas horas y crédito responden al modelo curricular propuesto. Sin embargo, no hay acuerdo en que existen especificaciones del peso asignado a cada área del currículo, así como también el nivel de aproximación entre el peso deseable establecido por el modelo para cada una de las áreas. En consecuencia, existe entre la muestra de estudio una relación fundamental entre la distribución correcta de las asignaturas, con horas y créditos, así como de las especificaciones de cada una en el desarrollo curricular. Eso puede ser un catalizador para aumentar la calidad educativa de la institución de educación superior.

Los resultados del equilibrio entre la teoría y la práctica se pueden observar en la Tabla 14. 
Tabla 14. Resultados sobre equilibrio entre la teoría y la práctica aplicada a la muestra de la Universidad Nacional de Ucayali.

\begin{tabular}{|c|c|c|c|c|c|c|}
\hline \multicolumn{7}{|c|}{$\begin{array}{l}\text { DESARROLLO CURRICULAR } \\
\text { Equilibrio entre la teoría y la práctica }\end{array}$} \\
\hline \multirow{2}{*}{$\mathbf{N}^{\circ}$} & \multirow{2}{*}{ PREGUNTAS } & TA & $\mathbf{A}$ & DU & D & TD \\
\hline & & $\%$ & $\%$ & $\%$ & $\%$ & $\%$ \\
\hline 39 & Especifica el énfasis teórico y práctico de cada asignatura o actividad. & 0 & 50 & 25 & 25 & 0 \\
\hline 40 & $\begin{array}{l}\text { Determina el número de horas teóricas y el número de horas prácticas de } \\
\text { cada asignatura o actividad en relación a los créditos signados. }\end{array}$ & 0 & 100 & 0 & 0 & 0 \\
\hline
\end{tabular}

Según la encuesta el $100 \%$ de la muestra está de acuerdo con que las horas de teoría y práctica concuerda con los créditos asignados a cada asignatura. El 50\% de los encuestados está de acuerdo con que se específica el énfasis teórico y práctico de cada asignatura o actividad. En este sentido, el desarrollo curricular muestra un equilibrio entre las horas de teoría y práctica para las asignaturas pertinentes por lo que muestra una fortaleza a la hora del desarrollo pedagógico. Esto incrementa los procesos de enseñanzaaprendizaje y aumenta el rendimiento académico de los estudiantes. Sin duda fortalece la calidad educativa de la institución y demuestra una buena gestión pedagógica.

En la Tabla 15 muestra los resultados del índice ponderado de prerrequisitos que se obtuvieron a través de la encuesta realizada a la muestra de la investigación.

Tabla 15. Resultados sobre el índice ponderado de prerrequisitos aplicada a la muestra de la Universidad Nacional de Ucayali.

\begin{tabular}{|c|c|c|c|c|c|c|}
\hline \multicolumn{7}{|c|}{$\begin{array}{c}\text { DESARROLLO CURRICULAR } \\
\text { Índice ponderado de prerrequisitos }\end{array}$} \\
\hline $\mathbf{N}^{\circ}$ & PREGUNTAS & $\begin{array}{l}\text { TA } \\
\%\end{array}$ & $\begin{array}{l}\mathrm{A} \\
\%\end{array}$ & $\begin{array}{l}\text { DU } \\
\%\end{array}$ & $\begin{array}{l}\mathrm{D} \\
\%\end{array}$ & $\begin{array}{l}\text { TD } \\
\%\end{array}$ \\
\hline 41 & $\begin{array}{l}\text { El número de materias preladas por semestre se encuentran en el rango } \\
\text { aceptable (mínimo } 25 \% \text { y máximo } 35 \% \text { ) }\end{array}$ & 0 & 25 & 75 & 0 & 0 \\
\hline 42 & Se han invitado las prelaciones innecesarias. & 0 & 25 & 75 & 0 & 0 \\
\hline 43 & Se presenta el flujo grama de prelaciones (malla curricular) & 0 & 0 & 0 & 100 & 0 \\
\hline
\end{tabular}


Según el 75\% de los encuestados mostraron dudas el número de materias preladas por semestre se encuentran en el rango aceptable, así como también en las prelaciones innecesarias. El $100 \%$ de la muestra manifiesta que no están de acuerdo con que la institución no presenta el flujograma de prelaciones, es decir la malla curricular. Todo esto se relaciona con que la universidad no toma como importante el desarrollo de la malla curricular y la importancia que tienen los prerrequisitos en la formación académica de los estudiantes. Es necesario que la gestión pedagógica desarrolle los mecanismos pertinentes en la formulación del pensum, esto genera la confianza en la masa estudiantil a la hora de poder inscribir las asignaturas en los lapsos académicos.

Los resultados de la racionalización del tiempo curricular manifestados por medio de la encuesta realizada a la muestra se detallan en la Tabla 16

Tabla 16. Resultados sobre racionalización del tiempo curricular aplicada a la muestra de la Universidad Nacional de Ucayali.

\begin{tabular}{|c|c|c|c|c|c|c|}
\hline \multicolumn{7}{|c|}{$\begin{array}{l}\text { DESARROLLO CURRICULAR } \\
\text { Equilibrio entre la teoría y la práctica }\end{array}$} \\
\hline $\mathbf{N}^{\circ}$ & PREGUNTAS & $\begin{array}{l}\text { TA } \\
\%\end{array}$ & $\begin{array}{l}\text { A } \\
\%\end{array}$ & $\begin{array}{l}\text { DU } \\
\%\end{array}$ & $\begin{array}{l}\text { D } \\
\%\end{array}$ & $\begin{array}{l}\text { TD } \\
\%\end{array}$ \\
\hline 44 & $\begin{array}{l}\text { Se especifica el trabajo docente, la preparación de clases prácticas y/o } \\
\text { actividades, la asesoría, consejería y los momentos para la investigación. }\end{array}$ & 0 & 50 & 50 & 0 & 0 \\
\hline 45 & $\begin{array}{l}\text { Establece la organización del currículo integral, cuadro lógico (secuencia } \\
\text { progresiva de los contenidos por áreas y especialidad. }\end{array}$ & 0 & 25 & 50 & 25 & 0 \\
\hline
\end{tabular}

No existe consenso entre los diferentes encuestados con respecto a la preparación del trabajo docente en la preparación de las actividades pedagógicas. Así como, el 50\% muestra dudas de que la universidad establece la organización del currículo integral donde muestra la secuencia de los contenidos por área y especialidad. La gestión pedagógica ha desarrollado un trabajo deficiente en el área docente a la hora de seguir la evaluación de la preparación docente con respecto a las clases que imparte en las diversas asignaturas. Del mismo modo, no hay un currículo integral donde las actividades se desarrollen en función de las pareas y contenidos programáticos.

En la Tabla 17 muestra los resultados de supervisión académica que se considera parte de la supervisión y control de calidad realizada a través de la encuesta realizada. 
Tabla 17. Resultados sobre supervisión académica aplicada a la muestra de la Universidad Nacional de Ucayali.

\begin{tabular}{|c|c|c|c|c|c|c|}
\hline \multicolumn{7}{|c|}{$\begin{array}{l}\text { SUPERVISIÓN Y CONTROL DE CALIDAD } \\
\text { Supervisión académica }\end{array}$} \\
\hline \multirow{2}{*}{$\mathbf{N}^{\circ}$} & \multirow{2}{*}{ PREGUNTAS } & $\mathbf{T A}$ & $\mathbf{A}$ & DU & D & TD \\
\hline & & $\%$ & $\%$ & $\%$ & $\%$ & $\%$ \\
\hline 46 & $\begin{array}{l}\text { Se ha previsto el asesoramiento técnico científico y didáctico para la } \\
\text { actualización. }\end{array}$ & 0 & 0 & 75 & 25 & 0 \\
\hline 47 & Existe disponible el cronograma de las actividades académicas. & 0 & 50 & 50 & 0 & 0 \\
\hline 48 & Existe un registro de seguimiento al estudiante y los egresados. & 0 & 0 & 50 & 25 & 25 \\
\hline 49 & $\begin{array}{l}\text { Se ha cumplido con el cronograma de capacitación a docentes y personal } \\
\text { administrativo }\end{array}$ & 0 & 0 & 50 & 25 & 25 \\
\hline
\end{tabular}

El $75 \%$ de los encuestados mostraron dudas con respecto que si la universidad ha prestado el asesoramiento técnico-científico y didáctico para la actualización. Hay un 50\% que manifestó que hay dudas en que existe un seguimiento de los estudiantes y egresados, así como también si ha cumplido con el cronograma de capacitación docente y al personal administrativo. Sin embargo, no hay consenso 9 en que si están disponibles los cronogramas de actividades académicas. Todo lo anterior manifiesta que la gestión pedagógica no está realizando la tarea en función del asesoramiento o capacitación al personal docente, administrativo técnico para las diferentes áreas que hacen vida dentro de la facultad. La supervisión académica es un parámetro fundamental para el desarrollo de la calidad educativa, debido a que sirve de medidor en el grado de satisfacción de los trabajadores universitarios y de los estudiantes. Permite corregir los problemas y avanzar hacia el progreso académico.

Los resultados de la pertinencia en la supervisión académica se pueden observar a través de la Tabla 18. 
Tabla 18. Resultados sobre pertinencia aplicada a la muestra de la Universidad Nacional de Ucayali.

\begin{tabular}{|c|c|c|c|c|c|c|}
\hline \multicolumn{7}{|c|}{ SUPERVISIÓN Y CONTROL DE CALIDAD } \\
\hline \multicolumn{7}{|c|}{ Pertinencia } \\
\hline $\mathbf{N}^{\circ}$ & PREGUNTAS & TA & A & DU & D & TD \\
\hline & PREGUNIAS & $\%$ & $\%$ & $\%$ & $\%$ & $\%$ \\
\hline 50 & $\begin{array}{l}\text { El modelo curricular está en concordancia con las necesidades y } \\
\text { expectativas de la comunidad. }\end{array}$ & 0 & 0 & 50 & 50 & 0 \\
\hline 51 & $\begin{array}{l}\text { Plantea acciones de desarrollo sostenible del sistema y los ecosistemas del } \\
\text { entorno. }\end{array}$ & 0 & 0 & 75 & 25 & 0 \\
\hline 52 & Las áreas del currículo corresponden al perfil que propone el modelo. & 0 & 0 & 75 & 25 & 0 \\
\hline 53 & $\begin{array}{l}\text { Existe relación entre el plan curricular y los ejes de interacción del modelo } \\
\text { propuesto. }\end{array}$ & 0 & 25 & 50 & 25 & 0 \\
\hline 54 & Existe aceptación del currículo desarrollado en el contexto. & 0 & 25 & 50 & 25 & 0 \\
\hline 55 & Existen políticas de colocación de los egresados en el mercado ocupacional. & 0 & 25 & 25 & 25 & 25 \\
\hline 56 & Los egresados tienen acogida en el mercado ocupacional. & 0 & 50 & 50 & 0 & 0 \\
\hline
\end{tabular}

Los resultados muestran que el $75 \%$ de los encuestados dudan con respecto a que la universidad planteara acciones de desarrollo sostenible del sistema y los ecosistemas del entorno y que las áreas del currículo corresponden al perfil que propone el modelo. Hay un $50 \%$ de dudas en función de la existencia de relación entre el plan curricular y los ejes de interacción del modelo propuesto, así como también dudas a la existencia de aceptación de currículo desarrollado en el contexto. Por otro lado, no hay consenso en relación al modelo curricular está en concordancia con las necesidad y expectativas de la comunidad, sobre las políticas de colocación de los egresados en el mercado ocupacional y que los egresados tienen acogida en el mercado ocupacional.

En definitiva, la universidad no persigue los fines del desarrollo de la calidad en la educación porque no busca información precisa de los egresados para poder conocer las fortalezas y debilidades del sistema académico y administrativo. Un aspecto importante que no se refleja es el compromiso con los Objetivos del Desarrollo Sostenible planteados por la Organización de las Naciones Unidad enfocadas en el período 2015-2030. Este es un paso para el desarrollo de una universidad vinculada con el respeto y resguardo de los ecosistemas, pero no solo en sus políticas internas sino en los mecánicos educativos para la formación de profesionales consientes de la grave crisis climática que vive el planeta. La universidad debe garantizar una gestión pedagógica enmarcada en esta situación. 


\section{CONCLUSIONES}

Luego de haber realizado el estudio de investigación y analizado los resultados obtenidos, concluimos con lo siguiente:

La gestión pedagógica se relaciona negativamente con la calidad educativa en la Facultad de Educación y Ciencias Sociales de la Universidad Nacional de Ucayali.

Se demostró que a través del programa estadístico EXCEL, con la prueba estadística la relación la gestión pedagógica se relación a significativamente con la calidad educativa en la Facultad de Educación y Ciencias Sociales de la Universidad Nacional de Ucayali

Según los resultados, se pudo analizar que los docentes muestran desinterés en aplicar y analizar los documentos pertinentes que contienen para el estudio amplio de la gestión pedagógica con la calidad educativa en la Facultad de Educación y Ciencias Sociales de la Universidad Nacional de Ucayali.

Finalizando, se observó que las autoridades no realizan o ejecutan de manera pertinente la gestión pedagógica para el desarrollo de una calidad educativa en la Facultad de Educación y Ciencias Sociales de la Universidad Nacional de Ucayali.

\section{REFERENCIAS}

Batista de los Ríos, D. (2017). Gestión pedagógica de la extensión universitaria para la formación integral del estudiante. Las Tunas: Tesis para optar al grado de Doctor en Ciencias Pedagógicas de la Universidad de Las Tunas. Obtenido de https://repositorio.uho.edu.cu/jspui/bitstr eam/uho/2426/1/tes.pdf

Bracho-Pérez, K. J. (2018). Modelo holístico de gestión pedagógica para la formación integral en la Universidad de Pamplona: análisis de una experiencia. Praxis, 14(2), 205-214. Obtenido de http://revistas.unimagdalena.edu.co/index .php/praxis/article/view/2672/2007
Calvo, J. F. (2018). Calidad educativa en la educación superior colombiana: una aproximación teórica. Sophia, 14(2), 4-14. Obtenido de https://dialnet.unirioja.es/servlet/articulo ?codigo $=6522053$

Chen-Quesada, E., Cerdas-Montano, V., \& Rosabal-Vitoria, S. (2020). Modelos de gestión pedagógica: Factores de participación, cambio e innovación en centros educativos costarricenses. Revista Electrónica Educare, 24(2), 317-345. Obtenido de https://www.scielo.sa.cr/pdf/ree/v24n2/ 1409-4258-ree-24-02-317.pdf

García Colina, F. J., Juárez Hernández, S. C., \& Salgado García, L. (2018). Gestión escolar y calidad educativa. Revista Cubana de Educación Superior, 37(2), 206-216. Obtenido de http://scielo.sld.cu/pdf/rces/v37n2/rces1 6218.pdf

Guano Romero, J. E. (2014). ESTRATEGIAS DE GESTIÓN EDUCATIVA DESDE LA PERSPECTIVA DE LA PRÁCTICA DE LIDERAZGO Y EL FORTALECER DEL BUEN VIVIR EN LA ESCUELA DE EDUCACIÓN BÁSICA NARCISO CERDA MALDONADO", DEL CANTÓN LA MANÁ, PROVINCIA DE COTOPAXI. Babahoyo, Ecuador: Trabajo de grado para optar al título de Magister en Docencia y Currículo de la Universidad Técnica de Babahoyo. Obtenido de http://dspace.utb.edu.ec/bitstream/handl e/49000/2069/MDC193.pdf?sequence $=1$ \&isAllowed $=y$

López-Paredes, M. A. (2017). La Gestión pedagógica. Apuntes para un estudio necesario. Dominio de las Ciencias, 3(1), 201-215. Obtenido de https://www.dominiodelasciencias.com/oj s/index.php/es/article/view/384

Palella Stracuzzi, S., \& Martins Pestana, F. (2010). Metodología de la investigación cuantitativa. Caracas, Venezuela: FEDUPEL, Fondo Editorial de la Universidad Pedagógica Experimental Libertador. 
Pareja, M. Q. (2020). La gestión pedagógica en la mejora del desempeño docente. Investigación valdizana, 14(1), 7-14. Obtenido de https://dialnet.unirioja.es/servlet/articulo ?codigo $=7409389$

Quintana-Torres, Y. E. (2018). Calidad educativa y gestión escolar: una relación dinámica. Educación y educadores, 21(2), 259-281. Obtenido de https://dialnet.unirioja.es/servlet/articulo ?codigo $=6718920$

Quispe Ramos, R. Y. (2018). Gestión pedagógica $y$ calidad educativa en instituciones educativas del nivel inicial de la Red 10UGEL 01, 2018. San Juan de Lurigancho, Perú: Trabajo de grado para optar al título de Mestra en Educación mención Docencia y Gestión Educativa de la Universidad Cesar Vallejo. Obtenido de https://repositorio.ucv.edu.pe/bitstream/ handle/20.500.12692/26875/Quispe_RR.p df?sequence $=1$ \&isAllowed $=y$

Rey, A. (2014). Gestión pedagógica del docente y dimensión axilógica del conocimiento. Dialógica: revista multidisciplinaria, 11(1), 111-132. Obtenido de https://dialnet.unirioja.es/servlet/articulo ?codigo $=5249543$

VISOR. (1999). Enciclopedia VISOR, tomo 12. Buenos Aires, Argentina: VISOR Enciclopedias Audiovisuales, S. A. 